\title{
HUBBLE SPACE TELESCOPE STUDIES OF NEARBY TYPE Ia SUPERNOVAE: THE MEAN MAXIMUM LIGHT ULTRAVIOLET SPECTRUM AND ITS DISPERSION
}

\author{
Jeff Cooke ${ }^{1}$, Richard S. Ellis ${ }^{1}$, Mark Sullivan $^{2}$, Peter Nugent $^{3}$, D. Andrew Howell ${ }^{4,5}$, Avishay Gal-Yam $^{6}$, \\ Chris Lidman $^{7}$, Joshua S. Bloom ${ }^{8}$, S. Bradley Cenko ${ }^{8}$, Mansi M. Kasliwal ${ }^{1}$, Shrinivas R. Kulkarni ${ }^{1}$, Nicholas M. Law ${ }^{9}$, \\ ERAN O. OFEK ${ }^{1,10}$, AND Robert M. Quimby ${ }^{1}$ \\ ${ }^{1}$ Cahill Center for Astrophysics, California Institute of Technology, Pasadena, CA 91125, USA; cooke@ astro.caltech.edu.edu \\ 2 Department of Astrophysics, University of Oxford, UK \\ ${ }^{3}$ Computational Cosmology Center, Lawrence Berkeley National Laboratory, Berkeley, CA 94720, USA \\ ${ }^{4}$ Las Cumbres Observatory Global Telescope Network, Goleta, CA 93117, USA \\ 5 Department of Physics, University of California, Santa Barbara, CA 93106-9530, USA \\ ${ }^{6}$ Astrophysics Group, Weizmann Institute of Science, Rehovot 76100, Israel \\ ${ }^{7}$ Australian Astronomical Observatory, Epping, NSW 1710, Australia \\ ${ }^{8}$ Department of Astronomy, University of California, Berkeley, CA 94720-3411, USA \\ ${ }^{9}$ Dunlap Institute for Astronomy and Astrophysics, University of Toronto, 50 St. George Street, Toronto M5S 3H4, Ontario, Canada \\ Received 2010 October 11; accepted 2010 December 6; published 2011 January 10
}

\begin{abstract}
We present the first results of an ongoing campaign using the STIS spectrograph on board the Hubble Space Telescope (HST), whose primary goal is the study of near-ultraviolet (UV) spectra of local Type Ia supernovae (SNe Ia). Using events identified by the Palomar Transient Factory and subsequently verified by ground-based spectroscopy, we demonstrate the ability to locate and classify SNe Ia as early as 16 days prior to maximum light. This enables us to trigger $H S T$ in a non-disruptive mode to obtain near UV spectra within a few days of maximum light for comparison with earlier equivalent ground-based spectroscopic campaigns conducted at intermediateredshifts, $\bar{z} \simeq 0.5$. We analyze the spectra of $12 \mathrm{SNe}$ Ia located in the Hubble flow with $0.01<z<0.08$. Although a fraction of our eventual sample, these data, together with archival data, already provide a substantial advance over that previously available. Restricting samples to those of similar phase and stretch, the mean UV spectrum agrees reasonably closely with that at intermediate redshift, although some differences are found in the metallic absorption features. A larger sample will determine whether these differences reflect possible biases or are a genuine evolutionary effect. Significantly, the wavelength-dependent dispersion, which is larger in the UV, follows similar trends to those observed at intermediate redshift and is driven, in part, by differences in the various metallic features. While the origin of the UV dispersion remains uncertain, our comparison suggests that it may reflect compositional variations among our sample rather than being predominantly an evolutionary effect.
\end{abstract}

Key words: cosmological parameters - supernovae: general - ultraviolet: general

\section{INTRODUCTION}

Type Ia supernovae (SNe Ia) remain the most practical and well-exploited cosmological probe offering an immediate route to understanding "dark energy." Measures of distant events are being used to distinguish between Einstein's cosmological constant, $\Lambda$, and a scalar field whose equation of state parameter $w \neq-1$ (Astier et al. 2006; Riess et al. 2007; Kessler et al. 2009; Amanullah et al. 2010). Yet despite remarkable observational progress, there is no satisfactory theory explaining an SN Ia event. The mechanism by which a white dwarf accretes additional material is unclear as is the nature of the explosion itself (Livio 2000).

To facilitate progress, observers employ a variety of empirical correlations to reduce the intrinsic scatter of the SN Ia Hubble diagram. SNe Ia were initially considered a one- or twoparameter family with the light curve width and rest-frame color as the key variables. However, improved data have revealed important correlations with the host galaxy. Events are not only more common in star-forming hosts per unit stellar mass but their light curve properties differ from those seen in quiescent galaxies (Sullivan et al. 2006, 2010), an effect that has direct consequences for their use over large look-back times (Howell et al. 2007).

\footnotetext{
${ }^{10}$ Einstein Fellow.
}

These discoveries naturally raise the question of what further evolutionary changes might be present in the SN Ia population. A long-standing concern has been the unknown effect of an evolving progenitor composition, both in terms of a possible redshift-dependent bias and in producing an intrinsic dispersion that could limit the effectiveness of large future surveys. A Keck study of high-quality rest-frame near-UV SNe Ia spectra at intermediate redshift $(z \simeq 0.5)$ reveals a surprising diversity at short wavelengths where some models predict a sensitivity to metallicity (Ellis et al. 2008, hereafter E08; see also Foley et al. 2008). A large $U$-band dispersion had earlier been claimed in the local photometric survey of Jha et al. (2006), although subsequent photometry has challenged the amount (Astier et al. 2006). Although some models predict that a UV dispersion might arise from variations in the progenitor composition (Lentz et al. 2000; Höflich et al. 2000), the magnitude of the effect seen by E08 exceeds that expected for reasonable compositional differences. Sauer et al. (2008) have shown a large fraction of the UV flux that can be formed by reverse-fluorescence scattering which affects the dependence on composition. If the observed dispersion is found to arise from some evolutionary trend, it could bias future $z>1$ SNe campaigns that typically sample from this wavelength region. Sullivan et al. (2009, hereafter S09) compare mean SN Ia spectra over a redshift path of $0<z<1.2$ and find no strong evolution; however, only three local UV spectra were available at the time, seriously 
limiting the comparison and giving no local measure of the UV dispersion.

Efforts to understand the UV behavior of $\mathrm{SNe}$ Ia have subsequently intensified. Using the UV optical telescope on board Swift, Brown et al. (2010) and Milne et al. (2010) have confirmed the presence of a dispersion increase to shorter wavelengths in local SNe Ia. As a result, comparisons between local and intermediate-redshift data remain unclear and so the question remains as to whether some component of the significant UV diversity seen in distant SNe Ia is an evolutionary phenomenon or represents some as yet unexplained diversity in the SN Ia mechanism.

As described in E08, spectroscopic studies offer a major advantage over photometric investigations as they eliminate uncertainties arising from $k$-corrections and, with adequate data, the mean and dispersion can be investigated in the context of known metallic features. Following the successful repair of the UV-capable Space Telescope Imaging Spectrograph (STIS) aboard the Hubble Space Telescope (HST) during the 2009 Servicing Mission 4, it has become possible to make significant progress in addressing the above questions. Using a non-disruptive Target of Opportunity (ToO) campaign (GO 11721, PI: Ellis), we are securing maximum light STIS spectra for $35 \mathrm{SNe}$ Ia located in the Hubble flow. Here, we present the results from the first 12 events from this program, augmented by three events from earlier archival data, that provide comparable statistics to the survey of E08 at intermediate redshift for the analysis undertaken here.

\section{OBSERVATIONS}

A significant challenge in delivering targets to $H S T$ for observations at maximum light is the need to detect and identify convincing $\mathrm{SNe}$ Ia candidates soon after explosion. For a non-disruptive ToO program, defined as one where Phase II observations are submitted for inclusion in the HST schedule built for the second week following the submission, targets must be identified $\sim 10-12$ calendar days prior to submission. As a result, spectroscopic SN Ia confirmations $\sim 7-16$ days before maximum light are necessary to acquire STIS observations with phases \pm 4 days from maximum light and match a distribution of intermediate redshift near UV spectra secured by the E08 campaign. Figure 1 illustrates the data acquisition timeline for the first $12 \mathrm{SNe}$ of our campaign that comprise the sample presented here-from detection, to ground-based spectroscopic confirmation, to STIS observation-and shows the ability of Palomar Transient Factory (PTF) to detect SN Ia outbursts as early as $\sim 16$ days prior to maximum light.

The identification of SNe Ia suitable for HST non-disruptive observations proceeded in two stages. The early identification of candidate events was based on photometric survey data, with 11 of the 12 newly discovered SNe identified between 2009 August 19 and 2010 June 29 by the PTF (Rau et al. 2009; Law et al. 2009). As with the CFHT Supernova Legacy Survey (SNLS; Astier et al. 2006) utilized by the E08 intermediate-redshift program, PTF is a rolling search for transient events unbiased to the nature of the host galaxy. The remaining event (SN 2009le; Pignata et al. 2009a) was triggered on the reported results of an independent search by the CHilean Automatic Supernova sEarch (CHASE; Pignata et al. 2009b) during a period when the Palomar observatory was under extensive ash clouds from the 2009 California forest fires.

Ground-based spectroscopic follow-up acquired within one to a few days after photometric detection represents the second

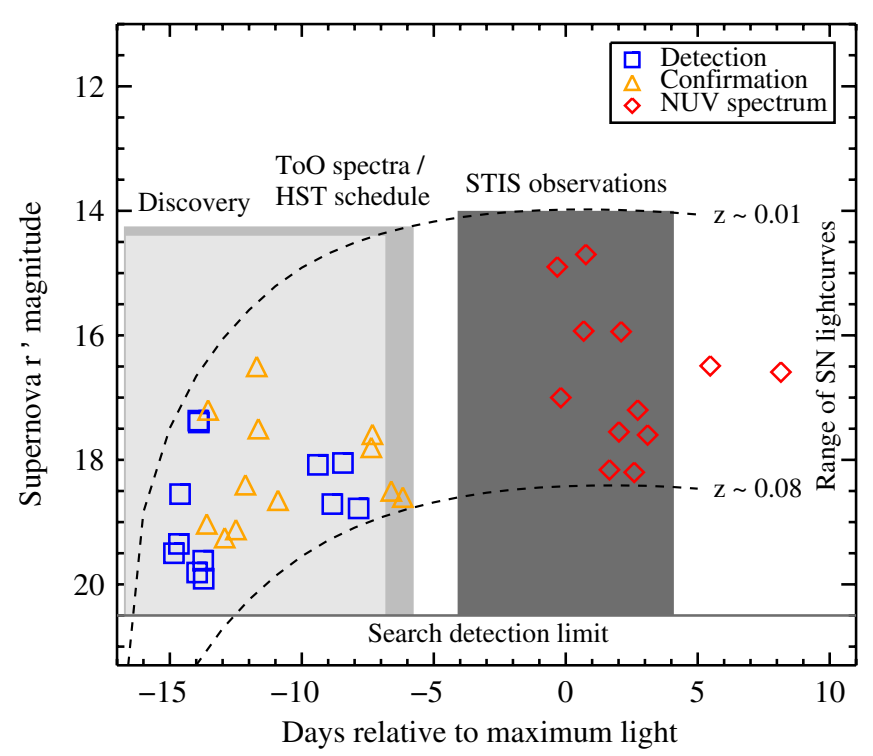

Figure 1. Timeline for the HST non-disruptive ToO program (GO 11721, PI: Ellis). The phase (number of days relative to maximum light) and approximate magnitude are shown for the PTF, and one non-PTF, photometric discoveries (squares), ground-based spectroscopic confirmations (triangles), and nearUV STIS spectra (diamonds). Photometric discoveries and spectroscopic confirmation are necessary during the time windows indicated by the lightgray and gray block regions, respectively, in order for STIS near UV spectra to be acquired within the time window indicated by the dark-gray region.

stage of confirmation essential for determining the SN type and redshift. Spectroscopy was performed using regularly scheduled time and occasional ToO interrupts on the following telescopes/instruments: the Low-Resolution Imaging Spectrometer (LRIS; Oke et al. 1995; McCarthy et al. 1998) and DEep Imaging Multi-Object Spectrograph (DEIMOS; Faber et al. 2003) on the Keck telescopes, the Gemini Multi-Object Spectrograph (GMOS; Hook et al. 2004) on the Gemini telescopes, the Focal Reducer and low-dispersion Spectrograph (FORS; Appenzeller et al. 1998) and X-shooter (Vernet et al. 2009) on the ESO Very Large Telescopes, the Double Spectrograph (DBSP; Oke $\&$ Gunn 1982) on the Palomar Hale Telescope, and the Intermediate dispersion Spectrograph and Imaging System (ISIS) on the William Herschel Telescope. By matching flux-calibrated spectra with selections from an extensive spectral database (Howell et al. 2005), improved phases typically accurate to \pm 2 days were obtained.

Near-UV spectra were acquired using the Space Telescope Imaging Spectrograph (STIS; Woodgate et al. 1998; Kimble et al. 1998) between 2009 September 02 and 2010 July 13 (Figure 1, red diamonds). To best match our $z \simeq 0.5 \mathrm{Keck}$ spectra (E08), we used the STIS/CCD 430L observing mode with 3-4 CR-SPLIT exposures totaling one orbit, ensuring good signal-to-noise ratio coverage down to a rest wavelength below 2900A. A montage of the STIS spectra is shown in Figure 2. Associated near-simultaneous ground-based optical spectra were also taken using further regularly scheduled time and $\mathrm{ToO}$ interrupts using the facilities described above. All data were reduced using standard IRAF and IDL data reduction routines. Pre- and post-maximum light PTF $r$-band photometric observations were used to determine the initial phase and stretch for each HST triggered event using the Si FT O light curve fitter (Conley et al. 2008). Details for the SN Ia sample here and the three archival SNe Ia (discussed in S09) are listed in Table 1.

In three cases (09dnl, 09dnp, 10fps), the PTF light curves are of marginal quality or too poorly sampled for an accurate 


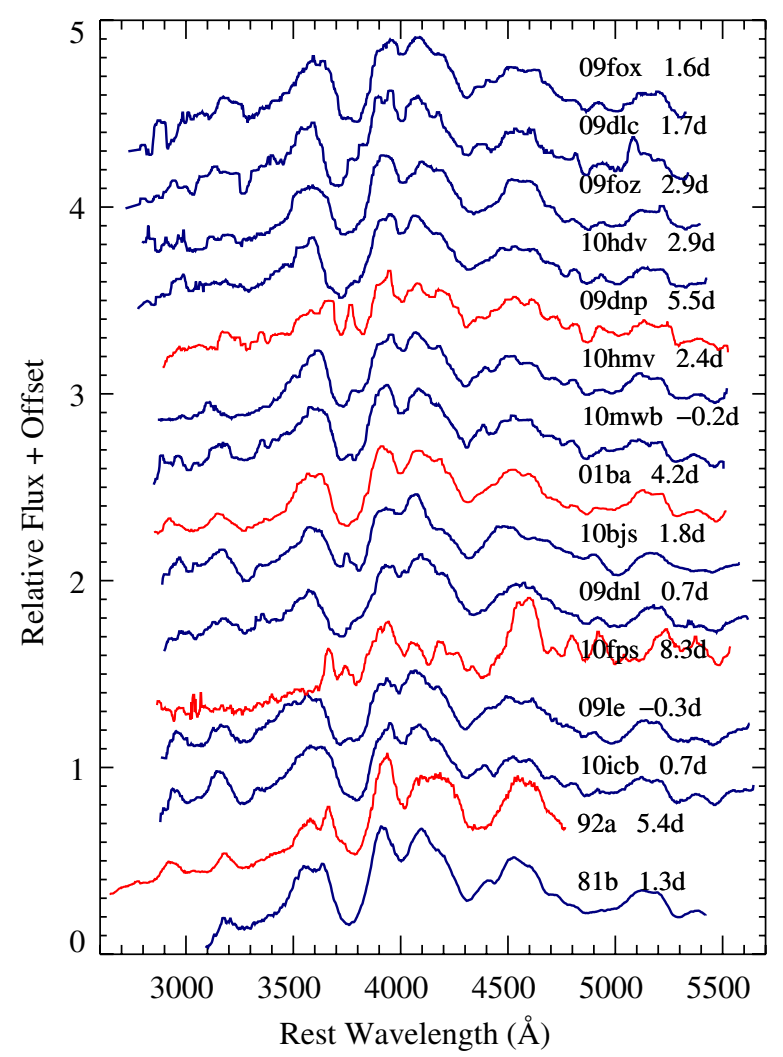

Figure 2. STIS near UV maximum-light spectra as itemized in Table 1 reduced to rest-frame wavelengths. Each label gives the PTF identification (with 09le indicating SN 2009le) and phase of STIS observations. Spectra in red represent those not included in the mean and dispersion analysis (see the text).

determination of one or both of the phase and stretch. One event (SN 2009le) has no PTF photometry and its phase was determined from the CHASE estimate (Challis \& Berlind 2009) which is consistent with our spectroscopic estimate. The sample we analyze below excludes four SNe (shown in red in Figure 2), 09dnp and 10fps and two archival SNe 92a and 01ba, based on their late phase or poor phase and/or stretch fits. We test this sample against a highly restricted sample (high phase and stretch fit quality with uncertainties of $<0.5$ and $<0.1$, respectively) and find the same trends and a negligible change to the results presented below.

\section{ANALYSIS}

Prior to this study, UV spectra were secured for 13 local $\mathrm{SNe}$ Ia, but only five were studied close to maximum light and two of these were peculiar. Suitable events from earlier work are listed in Table 1 and shown in Figure 2. In Cycle 13 a ToO campaign began to increase the sample (GO 10182, PI: Filippenko) but the failure of STIS curtailed this program. Remarkably, prior to our study, more was known about the premaximum and maximum light UV spectra of SNe Ia at $z \sim 0.5$ than at $z=0$. Our STIS sample has now dramatically improved this situation. Recently, Bufano et al. (2009) studied the UV spectra of three nearby non-peculiar SNe Ia using the Swift telescope. These are insightful data but, as the events are not located in the Hubble flow and at least one has insufficient light curve information for stretch measurement, they are less useful for the cosmological purpose explored here.

In order to compare the mean UV spectra of local SNe Ia and their dispersion with the sample discussed by E08, we must
Table 1

Low-redshift Supernova Ia Sample

\begin{tabular}{lccl}
\hline \hline Supernova & Phase $^{\mathrm{a}}$ & Stretch & \multicolumn{1}{c}{ Host $z$} \\
\hline PTF-09dlc & $+2.02 \pm 0.38$ & $1.152 \pm 0.054$ & 0.0675 \\
PTF-09dnl & $+0.68 \pm 1.99$ & $\ldots$ & 0.0231 \\
PTF-09dnp & $+5.47 \pm 0.87$ & $0.996 \pm 0.308$ & $0.0373^{\mathrm{c}, \mathrm{d}}$ \\
PTF-09fox & $+1.66 \pm 0.40$ & $1.016 \pm 0.109$ & 0.0718 \\
PTF-09foz & $+2.59 \pm 0.38$ & $0.883 \pm 0.079$ & $0.0543^{\mathrm{c}, \mathrm{d}}$ \\
PTF-10bjs & $+2.10 \pm 0.18$ & $1.138 \pm 0.019$ & $0.0300^{\mathrm{c}, \mathrm{d}}$ \\
PTF-10fps & $+8.15 \pm 4.85$ & $0.980 \pm 0.530$ & $0.0215^{\mathrm{c}, \mathrm{d}}$ \\
PTF-10hdv & $+3.10 \pm 0.36$ & $1.077 \pm 0.064$ & 0.0533 \\
PTF-10hmv & $+2.73 \pm 0.09$ & $1.150 \pm 0.009$ & 0.0324 \\
PTF-10icb & $+0.76 \pm 0.13$ & $1.071 \pm 0.021$ & $0.0086^{\mathrm{c}, \mathrm{d}}$ \\
PTF-10mwb & $-0.19 \pm 0.14$ & $0.896 \pm 0.018$ & 0.0313 \\
SN 2009le & $-0.32 \pm 1.38$ & $\ldots$ & $0.0178^{\mathrm{d}}$ \\
\hline SN 1981b & $+1.30 \pm 0.14$ & $0.89 \pm 0.02$ & $0.0060^{\mathrm{e}}$ \\
SN 1992a & $+5.45 \pm 0.04$ & $0.82 \pm 0.01$ & $0.0061^{\mathrm{e}}$ \\
SN 2001ba & $+4.21 \pm 0.22$ & $1.02 \pm 0.02$ & $0.0305^{\mathrm{e}}$ \\
\hline
\end{tabular}

Notes.

${ }^{a}$ Effective phase (phase/stretch) at the time of the STIS observations.

${ }^{\mathrm{b}}$ Unless otherwise noted, redshifts are obtained from the host features in the PTF spectra and are accurate to $z \lesssim 0.001$.

${ }^{c}$ Sloan Digital Sky Survey (SDSS).

d NASA Extragalactic Database (NED).

e Archival UV spectra discussed by S09 (Branch et al. 1983; Kirshner et al. 1993; Foley et al. 2008).

match the phase (and ideally the stretch) distributions of the two samples. Such a matched comparison was not possible in S09 due to the paucity of the local data. To maximize the utility of the new HST data, we adopt a phase range of -0.32 to +4 days (Figure 1) bounded by the earliest phase of the HST data $(-0.32)$ and a +4 day phase limit similar to that applied in E08 to minimize phase evolution effects on the mean and dispersion. This criterion leads to 11 events from the $H S T+$ archival sample drawn from Table 1 and 16 events from E08. Omitting the two events for which accurate stretches cannot be determined, the mean values are $S_{H S T}^{-}=1.030 \pm 0.038, \sigma=$ 0.114 and $S_{z \sim 0.5}=1.049 \pm 0.019, \sigma=0.076$. The two samples are consistent within the errors and have comparable distributions.

\subsection{Mean Type Ia Spectrum}

S09 presented a comparison of the mean UV spectra determined by E08 at $z \simeq 0.5$, those secured using the ACS grism by Riess et al. (2004) at $z \simeq 1.2$, and three local spectra from archival data listed in Table 1. Examining the $z \simeq 0.5 \mathrm{UV}$ spectra, S09 found some decrease with redshift in the strength of intermediate mass element features ( $\mathrm{Si}$ II, Ca II, and $\mathrm{Mg}$ II), but it was argued that this could arise in part due to the natural drift to luminous, larger stretch events expected at high redshift. Below $\lambda \simeq 3600 \AA$ the mean local spectrum was highly uncertain.

To facilitate a proper comparison to $\mathrm{S} 09$, we construct our mean near-UV spectrum following the procedure discussed in E08. Briefly, the spectra are normalized to have the same flux through a box filter defined between rest-frame 4000 and $4800 \AA$, and the variation in the mean spectrum is estimated via bootstrap resampling. Use of other box filters, including the full wavelength range common to all spectra, does not significantly affect the results. Figure 3 shows the normalized mean spectrum and region containing $90 \%$ of 100 bootstrapresampled mean spectra for both the low- and intermediateredshift matched samples. Although the low-redshift SN Ia mean 


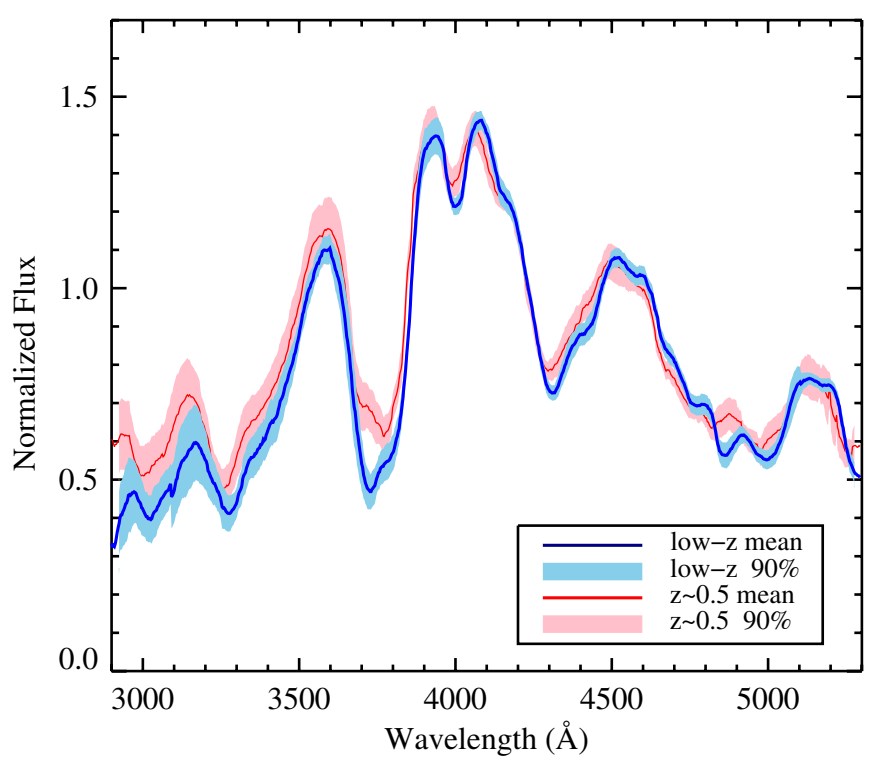

Figure 3. Mean near-UV spectra for low- and intermediate-redshift SN Ia near maximum light. The mean near-UV spectrum derived from the 10 STIS supernovae and one archival SN compliant with the adopted phase and stretch criteria (see the text) is shown by the blue line with the region containing $90 \%$ of the jack-knife resampling fits shown as the light-blue region. This is compared to the mean (red line) of a $z \simeq 0.5$ E08 sample of 16 SNe closely matched in phase and stretch and its $90 \%$ region (pink region).

spectrum closely resembles that at $z \simeq 0.5$, as in S09, we notice a marked decrease with increasing redshift in the depth of the Si II and Ca II blend near $3800 \AA, M g$ II near $4300 \AA$, and those of iron group elements below $3500 \AA$. The utility of the comparison is more advantageous than that conducted by S09 for wavelengths below $3500 \AA$ since our new local sample is much larger than that used by S09 and the phase and stretch distributions are better matched. Farther into the UV we note that the differences between the mean local and $z \simeq 0.5$ spectra become particularly significant. Since the stretch distributions of the two samples are similar, this could be a genuine effect rather than one arising from samples biased to more luminous and bluer events (Balland et al. 2009). Analysis of our eventual full sample will clarify this important point.

\subsection{Spectral Dispersion}

E08 demonstrated a significant increase in the variance of their $z \simeq 0.5$ SNe Ia UV spectra for wavelengths below $3700 \AA$, both by comparing individual deviations of 15 maximum light spectra from their mean in units of the dispersion $\sigma$, and via photometric colors measured directly from color-corrected Keck spectra. For the first time, our low-redshift sample of UV SN Ia spectra is large enough to perform a similar analysis. Ideally one would color-correct the local spectra following the procedure discussed in E08 using the SALT2 color law (Guy et al. 2007), but this requires host-corrected multi-color data that must await late-time reference images in bands other than the PTF $r$ band. We experimented with estimating the host contamination in $g$ and $i$ from the contemporary data but concluded that the uncertainties are too great at this time. Accordingly, a comparison with data from E08 that is not color-corrected is more appropriate. It is important to note that the color correction E08 applied did not significantly change the UV scatter and its wavelength dependent trend and we find this for the matched $z \sim 0.5$ sample as well. The key question we seek to address is whether the dispersion trend is generic to all SNe Ia, independent

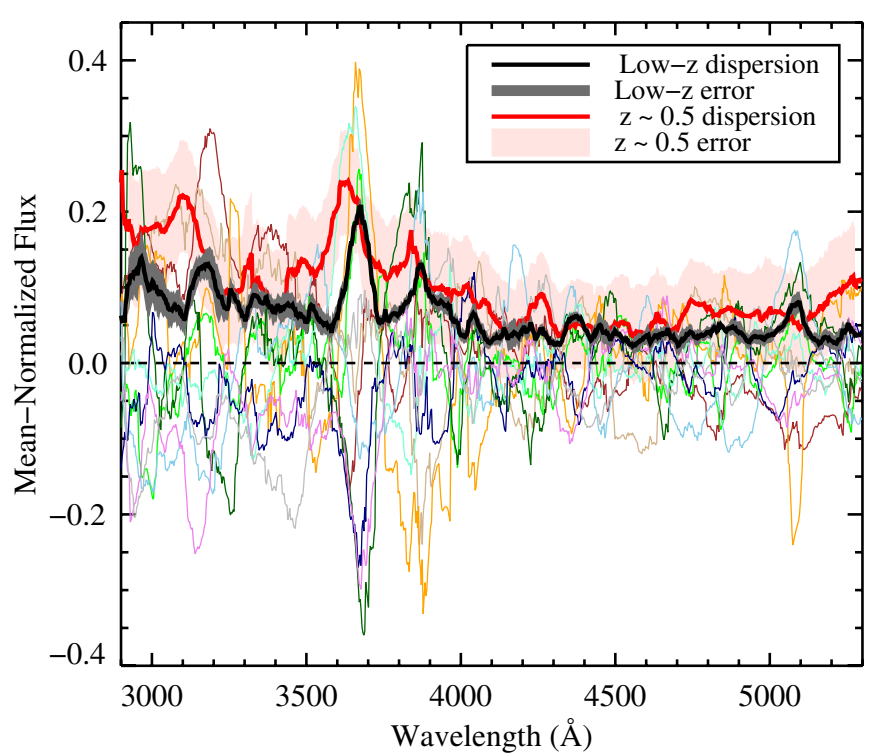

Figure 4. Dispersion from the mean for the 11 low-redshift $\mathrm{SNe}$ meeting our phase criteria (multi-colored thin curves). The absolute value of the mean dispersion is indicated by the thick black curve with $1 \sigma$ observational uncertainties overlaid (gray region). For direct comparison, the absolute value of the mean dispersion for the matched sample of 16 intermediate redshift E08 SNe is shown (thick red curve; uncertainties pink region). An increased dispersion at shorter wavelengths is present in both samples.

of redshift, or largely a feature of the intermediate redshift data only, possibly implying some evolutionary effect.

Figure 4 shows that the wavelength-dependent dispersion is indeed present in the local data, consistent with the photometric claims of Brown et al. (2010) and Milne et al. (2010). In the region that contains $90 \%$ of the mean values from bootstrap resampling, the variation from one spectrum to another with respect to the mean increases below $3700 \AA$ as in E08. Clearly several of the features which vary between the local and E08 samples discussed earlier contribute to the dispersion suggesting a compositional origin.

Even if we exclude the region dominated by strong features and consider the average dispersion from the mean spectrum in the regions UV: $2900-3500 \AA$ and optical: $4100-5200 \AA$ allowing for the spectrophotometric uncertainties, we find that the dispersion increases from the optical to the UV by a similar factor of $\simeq 2.3$ in both the local and $z \simeq 0.5$ samples. There is marginal evidence that the UV scatter may be larger in the $z \simeq$ 0.5 data than in the local sample, but the spectrophotometric uncertainties for the individual spectra are naturally larger. Overall, we conclude that the UV spectral dispersion is most likely a feature of the SNe Ia population and not an evolutionary effect.

\section{DISCUSSION}

Our initial results clarify and quantify indications from earlier work. Although the SN Ia mean spectrum close to maximum light appears to have remained remarkably similar over the past 5 Gyr, we find the decrease in the strength of the metallic features with increasing redshift noted by S09 also present in our more representative comparison. Given that the mean stretches of the local and $z \simeq 0.5$ samples are similar, this may represent the expected decrease in metallicity over this epoch. We can address this possibility in more detail with the completion of our survey.

Equally important is that we observe a strong wavelengthdependent scatter in the rest-frame UV spectra of our local 
sample, as noted in E08. Independent of the calibration questions that have plagued recent photometric studies, the spectra demonstrate that the UV scatter is generic to SNe Ia over a wide range of cosmic time and is not likely an evolutionary effect. Much of this behavior can be attributed to the varying absorption line strengths of intermediate mass elements occupying the UV wavelength region, supporting the notion that the UV scatter arises from compositional differences between events.

In addition to strengthening these conclusions with a larger sample, it is now clear that further progress will follow more detailed multi-phase UV studies of selected local events. HST has recently been awarded for such a program (GO 12298, PI: Ellis).

R.S.E. acknowledges support from DOE grant DESC0001101, M.S. from the Royal Society, A.G. from the Israeli Science Foundation and a European Union Marie Curie fellowship. Support for program GO 11721 was provided by NASA through a grant from the Space Telescope Science Institute, which is operated by AURA, Inc., under NASA Contract NAS5-26555. The National Energy Research Scientific Computing Center, which is supported by the Office of Science of the U.S. Department of Energy under Contract No. DE-AC0205CH11231, provided staff, computational resources, and data storage for this project. P.E.N. acknowledges support from the US Department of Energy Scientific Discovery through Advanced Computing program under contract DE-FG02-06ER0604. S.B.C. acknowledges generous support from Gary and Cynthia Bengier and the Richard and Rhoda Goldman Foundation.

\section{REFERENCES}

Amanullah, R., et al. 2010, ApJ, 716, 712

Appenzeller, I., et al. 1998, ESO Messenger, 94, 1
Astier, P., et al. 2006, A\&A, 447, 31

Balland, C., et al. 2009, A\&A, 507, 85

Branch, D., Lacy, C. H., McCall, M. L., Sutherland, P. G., Uomoto, A., Wheeler, J. C., \& Wills, B. J. 1983, ApJ, 270, 123

Brown, P., et al. 2010, ApJ, 721, 1608

Bufano, F., et al. 2009, ApJ, 700, 1456

Challis, P., \& Berlind, P. 2009, CBET, 2025, 1

Conley, A., et al. 2008, ApJ, 681, 482

Ellis, R. S., et al. 2008, ApJ, 674, 51

Faber, S. M., et al. 2003, Proc. SPIE, 4841, 1657

Foley, R. J., Filippenko, A. V., \& Jha, S. W. 2008, ApJ, 686, 117

Guy, J., et al. 2007, A\&A, 466, 11

Höflich, P., Nomoto, K., Umeda, H., \& Wheeler, J. C. 2000, ApJ, 528, 590

Hook, I. M., Jørgensen, I., Allington-Smith, J. R., Davies, R. L., Metcalfe, N., Murowinski, R. G., \& Crampton, D. 2004, PASP, 116, 425

Howell, D. A., Sullivan, M., Conley, A., \& Carlberg, R. 2007, ApJ, 667, L37

Howell, D. A., et al. 2005, ApJ, 634, 1190

Jha, S., et al. 2006, AJ, 131, 527

Kessler, R., et al. 2009, ApJS, 185, 32

Kimble, R. A., et al. 1998, ApJ, 492, L83

Kirshner, R. P., et al. 1993, ApJ, 415, 589

Law, N. M., et al. 2009, PASP, 121, 1395

Lentz, E. J., Baron, E., Branch, D., Hauschildt, P. H., \& Nugent, P. E. 2000, ApJ, 530, 966

Livio, M. 2000, in Type Ia Supernovae, Theory and Cosmology, ed. J. C. Niemeyer \& J. W. Truran (Cambridge: Cambridge Univ. Press), 33

McCarthy, J. K., et al. 1998, Proc. SPIE, 3355, 81

Milne, P. A., et al. 2010, ApJ, 721, 1627

Oke, J. B., \& Gunn, J. E. 1982, PASP, 94, 586

Oke, J. B., et al. 1995, PASP, 107, 375

Pignata, G., et al. 2009a, ATel, 2022, 1

Pignata, G., Maza, J., Hamuy, M., Antezana, R., \& Gonzales, L. 2009b, RevMexAA Conf. Ser., 35, 317

Rau, A., et al. 2009, PASP, 121, 1334

Riess, A. G., et al. 2004, ApJ, 600, L163

Riess, A. G., et al. 2007, ApJ, 659, 98

Sauer, D. N., et al. 2008, MNRAS, 391, 1605

Sullivan, M., Ellis, R. S., Howell, D. A., Riess, A., Nugent, P. E., \& Gal-Yam, A. 2009, ApJ, 693, L76

Sullivan, M., et al. 2006, ApJ, 648, 868

Sullivan, M., et al. 2010, MNRAS, 406, 782

Vernet, J., et al. 2010, Proc. SPIE, 7735, 50

Woodgate, B. E., et al. 1998, PASP, 110, 1183 